Nouvelles perspectives en sciences sociales

\title{
La longue marche de la sociologie relationnelle
}

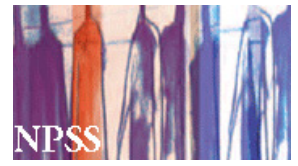

\section{Claude Vautier}

Volume 4, numéro 1, septembre 2008

URI : https://id.erudit.org/iderudit/019640ar

DOI : https://doi.org/10.7202/019640ar

Aller au sommaire du numéro

\section{Éditeur(s)}

Prise de parole

\section{ISSN}

1712-8307 (imprimé)

1918-7475 (numérique)

Découvrir la revue

\section{Citer cet article}

Vautier, C. (2008). La longue marche de la sociologie relationnelle. Nouvelles perspectives en sciences sociales, 4(1), 77-106. https://doi.org/10.7202/019640ar

\section{Résumé de l'article}

L'avenir de la sociologie est-il, comme l'imagine Monique Hirshhorn dans un texte publié en 2000, dans l'introduc-tion d'une « démarche proprement phénoménologique ", permettant de ne " pas réduire le "sujet" à l'acteur rationnel " et dans un nouveau questionnement « sur la construction sociale du soi » et " sur les modes d'élaboration de l'identité »? Ou bien s'agira-t-il plutôt de s'orienter vers une sociologie de la relation à laquelle appelaient notamment Laflamme en 1995, Emirbayer en 1997 ou Donati en 2004, au sein de réseaux comme dans l'analyse structurale ou dans une construction de modèles trialectiques proposée par Laflamme pour qui seule la relation, la communication, fonde le social et l'humain, est le social et l'humain? C'est cette question qui est au centre de cet article qui essaie de montrer que l'évolution contemporaine de la sociologie va d'une sociologie des "substances "

( " objets » ou " sujets ») vers une sociologie de la relation. 


\section{La longue marche de la sociologie relationnelle}

Claude Vautier

CIRESS-LEREPS, Université de Toulouse, Toulouse 1

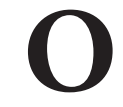

n connaît bien le vieux débat né des positions des précurseurs de la sociologie, dans lequel l'enjeu est de déterminer si ce sont les individus qu'il faut observer pour comprendre la structure sociale ou ces dernières qui contraignent les logiques d'action des individus au point que l'observation de ceux-ci ne serait d'aucune utilité pour l'étude sociologique.

La première position est celle initiée par Max Weber, pour qui " la sociologie, elle aussi, ne peut procéder que des actions d'un, de quelques, ou de plusieurs individus séparés. C'est pourquoi elle doit adopter des méthodes strictement individualistes ${ }^{1}$ ». La seconde l'a été par Émile Durkheim pour qui la structure sociale exerce un "déterminisme fort "sur l'individu qui n'agit qu'en fonction de cette détermination, ce qui impose une approche (ou une méthode) strictement holiste (bien que Raymond Boudon ait montré que Durkheim violait parfois sa propre règle en ce domaine ${ }^{2}$ ). Sans entrer dans le détail de la querelle entre indivi-

$1 \quad$ Max Weber, Lettre à R. Liefman le 9 mars 1920, cité dans Raymond Boudon et François Bourricaud, Dictionnaire critique de la sociologie, PUF, [1982] 1994, p. 1, article «Action».

2 Raymond Boudon, Études sur les sociologues classiques, Paris, PUF, 1998. Par exemple, «lorsqu'on examine non plus les écrits doctrinaux de Durkheim, 
dualisme méthodologique et holisme méthodologique, on peut remarquer qu'un premier pont a été jeté très vite entre ces deux positions par Georg Simmel :

les individus ne sont nullement les éléments derniers, les atomes du monde humain [...] En tant qu'elle se réalise progressivement, la société signifie toujours que les individus sont liés par des déterminations et des influences éprouvées réciproquement ${ }^{3}$.

Selon Pierpaolo Donati :

Longtemps demeuré implicite et comme en gestation, le " tournant relationnel " a constitué un thème central de la sociologie de Simmel [...] On peut considérer ce tournant relationnel comme l'acte de naissance d'une véritable théorie sociologique relationnelle .

On se trouve devant une lente, mais, nous semble-t-il, assez nette, évolution de la discipline sociologique : partie d'un conflit entre les tenants de la prééminence de la structure et ceux de la centralité du sujet autonome, la sociologie contemporaine en arrive à concevoir, en guise de dépassement, que le sujet fonde la structure qui, en retour, fonde le sujet. Cette évolution passe par des étapes connues : l'interactionnisme symbolique en fut une importante qui soulignait l'émergence du social des interactions entre les sujets ou les acteurs sociaux. L'individualisme méthodologique s'est développé dans une perspective similaire et

mais ses analyses elles-mêmes, on remarque que [...] Durkheim proclame une épistémologie empiriste, mais pratique une épistémologie non empiriste proche de celle de Weber ", dans le chapitre III, "Durkheim et Weber : convergence de méthode ", p. 101.

3 Georg Simmel (texte 1917), Sociologie et épistémologie, Paris, PUF, coll. "Sociologies ", 1981, cité par Alain Degenne et Michel Forsé, "Analyse de réseau et structuralisme ", dans Jean-Michel Berthelot (dir.), Sociologie. Épistémologie d'une discipline. Textes fondamentaux, de Boeck, 2000, p. 221234.

4 Voir Pierpaolo Donati, "La relation comme objet spécifique de la sociologie ", Revue du MAUSS, 2, n 24, 2004, p. 6, http://www.cairn.info/article_p. php?ID_ARTICLE=RDM_024_0233, site consulté le 25 septembre 2008. On s'accorde généralement à considérer que cette position de Simmel se trouve à la lointaine origine de la posture sociologique connue aujourd'hui en France sous les noms d'interactionnisme structural, d'individualisme structural, d'analyse structurale ou encore d'analyse de réseaux. On peut voir sur ce point Alain Degenne et Michel Forsé, Les réseaux sociaux. Une analyse structurale en sociologie, Paris, Armand Colin, coll. "U », 1994. 
Raymond Boudon écrit en 1979 que : "le sociologue [doit accorder] une attention constante à la complexité du jeu entre l'autonomie des agents et les contraintes des structures ${ }^{5} »$. De même, l'actionnisme de Michel Crozier et Erhard Friedberg tente de mettre en relief la relation existant entre des acteurs autonomes et des systèmes d'action qui influent sur les stratégies de ces acteurs. Bien d'autres auteurs, dont des sociologues majeurs comme Alain Touraine, pourraient être cités dans cette quête de la construction du social dans l'interaction entre l'acteur et le système, pour reprendre les termes de Crozier et Friedberg.

Et, cependant, cette évolution est chaotique dans la mesure où, selon des auteurs comme Pierpaolo Donati ou Simon Laflamme : «La plupart du temps, les sociologues n'en font pas un objet d'analyse, mais la résultante d'autre chose ${ }^{6} »$. Ainsi, Guy Bajoit, auteur d'un ouvrage intitulé Pour une sociologie relationnelle ${ }^{7}$, mais aussi Contribution à une sociologie du sujet ${ }^{8}$, écrit-il, dans un autre ouvrage : " La sociologie ne peut comprendre la vie sociale d'aujourd'hui qu'en plaçant le sujet individuel au cœur de son approche ${ }^{9}$. Donati et Laflamme considèrent, pour leur part, que l'objet de la sociologie ne peut être le sujet mais les relations, comprises comme la catégorie centrale de cette discipline. Ils affirment que "La société est relations (et non qu'elle a des relations) ${ }^{10}$ ", que, " cela n'implique pas non plus de se tourner vers une forme d'ontologie niant l'humain ou le sujet social " et que " la relation n'élimine pas les termes qu'elle met en connexion; au contraire, elle les met en valeur, les explore, les exprime $^{11} "$.

L'objet de ce texte n'étant pas d'effectuer une recension des

5 Raymond Boudon, La logique du social. Introduction à l'analyse sociologique, Paris, Hachette 1979, p. 296.

6 Pierpaolo Donati, "La relation comme objet... ", op. cit., p. 3.

7 Guy Bajoit, Pour une sociologie relationnelle, Paris, PUF, 1992.

8 Guy Bajoit, Contribution à une sociologie du sujet, Paris, L'Harmattan, 2000.

9 Guy Bajoit, Le changement social. Approche des sociétés occidentales contemporaines, Paris, Armand Colin, coll. "Cursus », 2003.

10 Pierpaolo Donati, "La relation comme objet... ", op. cit., p. 7.

11 Ibid., p. 17 et 19. 
théories de l'acteur ou de la relation, mais d'envisager comment la sociologie a traité et pourrait traiter cette question, nous nous contenterons d'évoquer quelques-unes des bornes de cette histoire, en partant de l'interactionnisme symbolique pour aller vers l'interactionnisme structural (ou analyse structurale ou analyse de réseau) pour aboutir à la sociologie relationnelle proposée par Pierpaolo Donati, en Europe, Mustafa Emirbayer, aux ÉtatsUnis, et par Simon Laflamme, au Canada.

À travers ce parcours, nous essaierons de montrer que les tentatives de promouvoir une sociologie relationnelle semblent buter sur cette difficulté particulière que Laflamme, de son côté, énonce ainsi :

Il ne suffit plus aux sciences humaines contemporaines de répéter plus ou moins gauchement que l'humain est déterminé et déterminant, il faut maintenant tirer toutes les conséquences de cette importante conclusion. Et l'on ne peut le faire qu'en faisant de l'humain lui-même le lieu de la dialectique ${ }^{12}$.

\section{1. Évocation rapide de l'histoire de la « sociologie des relations »}

Nous nous limiterons à des positions exemplaires, l'interactionnisme, l'individualisme méthodologique, l'analyse structurale et la sociologie relationnelle, à travers un nombre limité d'auteurs, ceux que nous considérons comme ayant joué un rôle de premier plan dans le développement de la position qu'ils défendaient ou défendent actuellement.

\subsection{Interactionnisme}

L'histoire de cette approche passe par de nombreux apports parmi lesquels ceux des Écoles de Chicago qui développèrent, dès 1892 (ouverture de l'Université de Chicago), un modèle de sociologie interactionnelle qui se cristallisa en interactionnisme symbolique et ethnométhodologie. Dans son principe, l'interactionnisme pose que la structure sociale est un effet émergent des interactions

12 Simon Laflamme, Communication et émotion. Essai de microsociologie relationnelle, Paris, L'Harmattan, coll. "Logiques sociales ", 1995, p. 143. 
entre les individus, ce qui apparaît comme une proposition tout à fait acceptable pour nous. Mais, en contrepartie, il a pu essuyer la critique (Habermas, Crozier, Friedberg...) de se focaliser sur la construction subjective que font les acteurs de leur monde, sans référence réelle à des normes sociales qui n'y apparaissent que comme des constructions subjectives de sens, des ressentis purs (stigmatisation, étiquetage).

En prolongeant cette interprétation et en la radicalisant, l'ethnométhodologie apporte un éclairage sur « la construction sociale de la réalité " par les individus en interaction, souligne leur compétence quasi universelle à comprendre les phénomènes sociaux dont ils sont partiellement créateurs et gomme la frontière entre connaissance profane et connaissance savante, par l'application d'un "sens pratique " (les "méthodes » ou " ethnométhodes ») qui leur permet de régler les problèmes de leur vie quotidienne.

Globalement, l'une et l'autre de ces deux positions ont exploré l'idée essentielle selon laquelle la structure sociale n'est qu'une émergence des interactions entre les individus et celle, non moins essentielle, qui est que le monde social est aussi lié à l'émergence d'un sens produit inter-individuellement et partagé.

Cependant, on a pu leur reprocher (en particulier en ce qui concerne l'ethnométhodologie) de s'être essentiellement focalisées sur l'interaction : c'est presque dans un face à face entre deux individus que se joue la vie sociale, à travers un langage commun et la réalité sociale ne serait plus que ce qu'en disent les acteurs. Les aspects institutionnels, les normes et valeurs partagées (quand bien même elles sont produites par les individus en interaction, mais aussi intériorisées et pas seulement dans une situation précise dans l'espace et le temps) deviennent singulièrement absents.

Et, cependant, la critique elle-même n'est pas exempte de reproche. En insistant sur l'absence des normes intériorisées par les acteurs, celle-ci renvoie à une position dans laquelle les acteurs deviennent (ou restent) le centre de l'analyse. Ainsi que l'écrivent Alain Degenne et Michel Forsé : 
Parti d'un dispositif supposant l'indépendance des unités d'analyse, on en arrive très logiquement à des explications psychologisantes : les individus qui ont le même comportement partagent les mêmes normes ou ont la même conscience collective, qui les poussent à agir de la même façon. En un mot, les normes sont des causes. Le point de vue de l'analyse structurale est très exactement inverse. Les normes sont des effets de la situation structurale des individus ou des groupes. [...] Il est donc possible de se passer du recours aux explications selon lesquelles les acteurs sont mus par des forces (des normes intériorisées, des forces collectives ou plus récemment des habitus), c'est-à-dire en fin de compte d'un recours à une causalité abstraite synonyme d'un âge métaphysique pour reprendre le vocabulaire de Comte ${ }^{13}$.

Il ne paraît pas excessif de penser que les courants de pensée interactionnistes ${ }^{14}$, dans leur développement même, en se recentrant sur une communication entre individus ayant des compétences dans les situations qu'ils vivent, avec une rationalité implicite qui devient le principe sous-jacent de toute interaction sociale, restent très éloignés de ce qu'ils auraient pu apporter. Dans son Manifesto publié en 1997, Mustafa Emirbayer pointe le fait que l'interactionnisme reste un substantialisme, dans la mesure où il s'intéresse au mieux à l'interaction de deux entités fixed, preconstituted et self-subsistent. Ce n'est pas au réseau que s'intéresse l'interactionnisme, c'est aux individus qui sont préalables à la relation (ou à l'interaction), individus dont les caractéristiques essentielles ne sont pas fondamentalement modifiées par cette relation ${ }^{15}$. Et, reprenant Margaret Somers et Gloria Gibson, qu'il cite, Emirbayer affirme par la médiation de ces auteures : "The classification of an actor divorced from analytic relationality is neither ontologically intelligible nor meaningful ${ }^{16}$ ".

Loin de développer une possible synthèse, entre approche holiste et individualiste, voire un dépassement de ces deux positions, les interactionnistes ne perçoivent pas l'idée selon

13 Alain Degenne et Michel Forsé, Les réseaux sociaux..., op. cit., p. 6-7.

14 De même que leurs critiques, d'ailleurs, qui insistent sur la question des normes intériorisées par ces individus et, par cela, recentrent l'analyse soit sur l'acteur, soit sur le système.

15 Mustafa Emirbayer, "Manifesto for a Relational Sociology ", American Journal of Sociology, vol. 103, $\mathrm{n}^{\circ} 2$, 1997, p. 281-317.

16 Ibid., p. 288. 
laquelle l'acteur est le système comme le système est la production des acteurs : ils coupent la boucle entre les deux termes de l'analyse ${ }^{17}$. Ils ne perçoivent pas non plus que la relation peut être conçue comme une catégorie centrale de l'analyse sociologique : pour eux, la relation est avant tout " relation entre des choses " et c'est à ces " choses " qu'ils s'intéressent.

\subsection{Individualisme méthodologique et actionnisme $e^{18}$}

Pour ses promoteurs principaux, Raymond Boudon et François Bourricaud, l'individualisme méthodologique permet de comprendre le social à travers les actions des humains développant des intensions et des interrelations : "Une explication est dite individualiste (au sens méthodologique) lorsque l'on fait explicitement de $\mathrm{P}$ (un phénomène social $\mathrm{P}-N D A$ ) la conséquence du comportement des individus appartenant au système social dans lequel $\mathrm{P}$ est observé ${ }^{19}$ ». Un système social est constitué de volontés individuelles en action, en lutte-coopération, comme chez Crozier et Friedberg, qui s'agrègent pour donner naissance à ces régularités de phénomènes que l’on peut considérer comme structurelles et que Simmel appelle des « formes». Comprendre ces structures, c'est, pour l'individualisme méthodologique, comprendre les motivations qui ont poussé les acteurs à agir ainsi et non autrement. Et cette compréhension suppose que l'on se dote d'une "psychologie de convention " (terme emprunté à Simmel), autrement dit d'un modèle de rationalité (en l'occurrence, ce sera une rationalité large - « sémantique » dit Monique

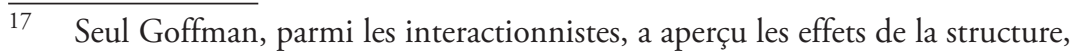
mais sur le tard, et jamais en concevant une perspective dialectique. Ainsi, écrit Mustafa Emibayer : "To the extent that they had been theorized, face-toface encounters were most typically seen (at least prior to Erving Goffman) in self-actional or in interactional terms, as a question of the mutual interplay among precontituted, self-subsistent actor", ibid., p. 295.

18 Monique Hirshhorn distingue l'actionnisme de l'individualisme méthodologique en présentant ce dernier comme « le premier principe du paradigme actionniste ", ("L'actionnisme ", dans Jean-Michel Berthelot (dir.), La sociologie française contemporaine, Paris, Presses universitaires de France, [2000] 2001, p. 48).

19 Raymond Boudon et François Bourricaud, Dictionnaire critique de la sociologie, PUF, [1982] 1994, p. 306. 
Hirshhorn ${ }^{20}$, correspondant à l'idée qu'est rationnelle toute action que l'acteur avait de bonnes raisons d'accomplir -, non seulement utilitariste, mais aussi limitée - bounded rationality de Simon - et axiologique, au sens de Weber).

Lindividualisme méthodologique représente la société comme un système au sens d'un " complexe d'éléments en interaction ", si l'on reprend la définition la plus simple, celle de Ludwin von Bertalanffy ${ }^{21}$. C'est même "un système d'interdépendance ${ }^{22}$ " dont parfois " la structure est telle qu'ils (les acteurs du système $N D A$ ) sont incités à produire un résultat qu'ils ne recherchaient certainement pas $^{23} "$.

On voit bien, à travers ces quelques considérations centrales dans l'œuvre de Boudon, combien ce positionnement se concentre sur l'acteur, sa rationalité et son intentionnalité. On voit également que ce paradigme prête attention à l'interaction dont se construisent les configurations propres à développer des effets de composition ou des effets pervers. On y retrouve les caractéristiques du courant interactionniste. Mais on y retrouve également les limites de celui-ci : centration sur l'acteur, nécessité de poser un principe de rationalité (même large) et une intentionnalité de l'acteur, c'est-à-dire d'être attentif à sa psyché pour comprendre ses comportements créateurs du Tout social. Les individus, les " agents ", sont certes producteurs du social et le social modifie leur comportement selon le contexte, mais ils sont cependant prédéterminés par une rationalité a priori et une intentionnalité initiale (ils n'obtiennent pas forcément ce qu'ils recherchaient, car ils recherchaient forcément quelque chose...). Lanalyse reste fixée sur cet élément central qu'est l'agent et, là encore, la boucle est coupée entre l'acteur et le système et la relation n'est, d'une certaine manière, qu'un médium entre des

\footnotetext{
20 Monique Hirshhorn, "L'actionnisme ", op. cit., p. 50.

21 Ludwin von Bertalanffy, Théorie générale des systèmes, Paris, Dunod, 1968, p. 53.

22 "Les systèmes d'interdépendance sont souvent caractérisés par le fait que les actions émises par les agents du système engendrent des phénomènes collectifs en tant que tels non voulus par ces agents " (Raymond Boudon), La logique du social..., op. cit., 1979, p. 118.

Ibid., p. 123.
} 
agents intentionnels.

\subsection{Analyse structurale ou analyse de réseaux}

En s'opposant aux « explications psychologisantes », l'analyse structurale referme la boucle d'une certaine façon.

Les réseaux sociaux ne sont pas constitués de " tuyaux " reliant des identités figées. Ils résultent des efforts de contrôle des identités pour faire face à l'incertitude radicale, aussi bien dans le monde social que dans le monde bio-physique. Ces efforts de contrôle produisent des histoires dont dérivent les liens qui constituent les réseaux [...] La notion de réseau qui émerge de cette approche est étroitement liée à de complexes formations narratives et peut servir aussi bien aux acteurs qu'aux observateurs pour rendre compte de la vie sociale ${ }^{24}$.

Ce faisant, l'analyse de réseaux réintroduit également la temporalité là où elle avait été occultée.

Les structures de relation résultent aussi de processus inscrits dans le temps, et [...] sans une telle description, les analyses s'égarent dans des explications culturelles et fonctionnalistes, les deux faisant habituellement leur apparition lorsque les dynamiques historiques ont été négligées ${ }^{25}$.

Plus généralement, affirmant « la structure comme contrainte et effet émergent ", Degenne et Forsé soulignent la nécessité posée par l'analyse structurale de passer " des catégories aux relations " :

La plupart des sociologues admettent que les comportements ou les opinions des individus dépendent des structures dans lesquelles ils s'insèrent. Pourtant, au lieu de penser la réalité en termes de relations, beaucoup de ceux qui traitent de données empiriques la pensent en termes de catégories (par exemple, les jeunes, les femmes, les cadres, les pays en voie de développement, etc. $)^{26}$.

L'insistance sur l'approche par la relation plutôt que par les

24 Harrison White, cité par Michel Grossetti et Frédéric Godart, "Réseaux et histoires ", Sociologies, 17 octobre 2007, p. 1, http://sociologies.revues.org/ document 240.html, site consulté le 25092008.

25 Ronald L. Breiger, cité par Michel Grossetti et Frédéric Godart, op. cit., p. 5.

26 Alain Degenne et Michel Forsé, Les réseaux sociaux..., op. cit., p. 5. On peut remarquer que Donati et Laflamme considèrent la ou les relation(s) comme une nouvelle catégorie susceptible de remplacer avantageusement les autres de nature substantives (acteur, classe sociale, cadres...) pour la compréhension des ensembles sociaux. 
catégories ouvre un important champ d'investigation à la sociologie contemporaine et lui fournit de nouveaux outils d'une grande puissance. D'une part, elle recoupe les approches systémiques et systémiques complexes développées parallèlement et depuis le début du XX $\mathrm{XX}^{\mathrm{e}}$ siècle, d'autre part, elle développe des théories formelles et des méthodes empiriques, telles celles des graphes, des hypergraphes ou des treillis de Galois, celles de la reconstruction $^{27}$ ou encore les systèmes multi agents centrés acteur ou centrés système. Enfin, son inscription dans ce domaine interdisciplinaire qu'on nomme sociologie économique lui permet d'aborder un large éventail de problèmes concrets qui se posent aux sociétés contemporaines. Ce qui fait écrire à Emirbayer : " the best developed and most widely used approaches to the analysis of social structure are clearly those of social-network analysis ${ }^{28}$ ".

\section{Approche relationnelle et communicationnelle de Laflamme}

Nous aurions pu partir de la position de celui que l'on appelle parfois le père de la sociologie relationnelle, Pierpaolo Donati ou de celle du représentant sans doute le plus connu de cette orientation, Mustafa Emirbayer. Mais, considérant que les trois approches ont des points communs très nombreux, nous allons directement vers l'approche communicationnelle du sociologue canadien, sans doute moins médiatique et moins connue, mais sans doute aussi plus radicale.

Dans la sociologie de Simon Laflamme, le point de départ est la relation. Et la relation est communication ${ }^{29}$.

De même que dans les analyses sociologiques qui ont été évoquées plus haut, dans les approches communicationnelles les plus courantes, en psychologie, en sociologie ou dans le domaine des relations industrielles, la communication est supposée s'établir en extériorité entre deux ou plusieurs acteurs sociaux préexistants

27 Camille Roth, «Reconstruction en sciences sociales : le cas des communautés de savoir ", Nouvelles perspectives en sciences sociales, vol. 2, $\mathrm{n}^{\circ} 2,2007$, p. 59-101.

28 Mustafa Emirbayer, "Manifesto for a Relational Sociology ", op. cit., p. 298.

29 Voir la section : "Relation et communication : deux concepts identiques? ". 
à la relation, intentionnels et rationnels ${ }^{30}$. Ces acteurs sont conscients parce qu'intentionnels, intéressés parce que rationnels.

Ces approches, au-delà des éclairages pertinents qu'elles ont pu apporter à l'étude de la communication, laissent échapper quelque chose qui correspond à un fondement majeur des sciences de la communication et, plus généralement, des sciences sociales.

Ce " quelque chose " peut être traduit en opérant un renversement de la tradition de pensée en ce domaine : la communication n'est pas un flux passant d'un agent communicant à un autre, le flux pouvant s'écouler ou non, selon que la communication est établie ou ne l'est pas. La communication est toujours déjà là, constitutive de l'être social.

Admettons que relation et communication soient deux choses différentes. Que, dans ce cas, la communication peut, d'une certaine manière, être coupée; la relation, pourtant, est toujours là, même si la communication semble être un ensemble vide. Mais une telle séparation entre "relation " et " communication " n'apporte sans doute rien d'autre que la réintroduction de l'idée que la communication est un acte attaché à l'intentionnalité du sujet humain (il peut décider de communiquer ou non), tandis que la relation ne le serait pas (même en le souhaitant, l'être humain est pris dans un faisceau de relations sur lesquelles sa volonté a peu de poids), écartant ainsi l'idée même d'une relation et d'une communication toujours là à l'œuvre dans le social, constitutive de l'être toujours social. Ce qui conduit dans ce cas, écrit Simon Laflamme, à ce que :

L'étude porte sur les pôles de la relation et non sur ce qui se réalise en elle. C'est l'effet ou la cause qui préoccupe et non le lieu qui les rend possibles, étant entendu que la relation est a priori déterminée par la

$\overline{30}$ Les questions de la rationalité et de l'intentionnalité ne sont pas étudiées ici en tant que telles. Mais elles interviennent comme des catégories liées à une approche husserlienne de type phénoménologique, à la notion de " conscience ", renvoyant à un acteur constituant le centre d'une sociologie où ce sont les caractéristiques de ce dernier qui expliquent les mouvements du social, bien entendu en interaction, mais séparé du système de relations complexes dont il est le sujet en même temps que l'objet. 
nature des consciences ou qu'elle n'a pour effet que de les naturali$\operatorname{ser}^{31}$.

Alors que :

En réalité, il n’y a ni pensée ni émotion dans le simple champ du sujet puisque le sujet est toujours un être déjà relationnel, puisque sa pensée et ses émotions ne peuvent se manifester en dehors du champ de la communication $^{32}$.

Dans ce qui suit, les termes "relation » et " communication" ne seront plus disjoints : on considèrera qu'être en relation, c'est être en communication, non seulement éventuellement intentionnelle, gestuelle, verbale, etc., mais aussi, non intentionnellement, du fait d'une simple co-présence au monde.

\subsection{Observer le système de communication et non la communication elle-même}

Dans cette approche relationnelle appliquée à des questions microsociologiques, l'adoption d'un angle de vue si radicalement différent interdit de continuer à se représenter empiriquement le niveau ou le degré d'une relation (ou d'un ensemble de relations) en mesurant le seul degré de satisfaction des agents communicants. Il ne s'agit plus de s'attacher au seul acteur ou agent, à ses ressentis (satisfaction), mais aussi au système de relations luimême. En caractérisant ce système, il est alors possible de déterminer en quoi sa configuration ${ }^{33}$ intervient dans les résultats de la communication entre divers acteurs sociaux. C'est désormais le système qu'il s'agit de spécifier.

De fait, si l'on considère que le système de communication est

31 Simon Laflamme, Communication et émotion..., op. cit., p. 28.

32 Ibid., p. 32.

33 Le concept de " configuration " apparaît chez Norbert Elias dans La société des individus (Paris, Fayard, [1987] 1991), et est relevé par Roger Chartier dans son avant-propos, p. 12 et p. 14. Il peut être explicité par cette citation d'Elias disant qu'à l'aide de ce concept : "on peut desserrer la contrainte sociale qui nous oblige à penser et à parler comme si l'" individu » et la "société " étaient deux figures différentes et de surcroît antagonistes " (op. cit., p. 14). Ce concept est proche de celui utilisé par l'analyse structurale qui tente de faire apparaître une topographie, une histoire et une appréciation de la force de liens existant entre individus dans un réseau. 
toujours déjà là, les émotions comme la raison des individus émergent de cette situation de communication, de la conjonction de tous les « être-là » en relation.

On tient généralement pour évident que les émotions prennent leur origine dans le psychisme de l'individu et qu'elles y ont là leur siège. Pourtant, cette opinion est loin d'être incontestable, car elle omet de prendre en considération une réalité profonde : le fait que l'humanité vit essentiellement au sein de relations. Le fondement de l'humanité, ce n'est pas la subjectivité mais la relation historicisée. Les émotions mêmes sont le produit des relations humaines et non des humains en tant que sujet $^{34}$.

Cette déclaration, qui présente la thèse développée dans l'essai, peut être, nous semble-t-il, rapprochée de ce que disait Bateson au cours d'une discussion avec Henryk Skolymoski et quelques autres chercheurs, à Darlington Hall, en Angleterre, en octobre 1979 :

Lorsqu'on fait un peu de méditation, on découvre avec surprise la différence entre penser aux choses et penser aux relations entre les choses [...] Une hache n'a pas d'utilité. L'utilité d'une hache est liée à sa position entre un homme et un arbre [...] demander " quel est le sens de l'univers? " implique qu'il existe une autre entité pour laquelle l'univers aurait un sens. Le sens n'est pas interne. Il émerge de la relation entre les parties ${ }^{35}$.

De même, chez Alex Mucchielli, peut-on lire que :

Le sens naît de la mise en relation de l'action de communication avec les éléments de son environnement [...] Les systèmes symboliques que les gens utilisent pour construire des significations sont déjà en place : ils sont déjà làn.

Cependant, chez Bateson, Mucchielli et de nombreux autres auteurs traitant de la communication (Watzlawick, par exemple), on a le sentiment que cet apport de la dimension relationnelle garde quelque chose de l'ancien paradigme : la relation est tou-

$\overline{34}$ Simon Laflamme, Communication et émotions..., op. cit., quatrième de couverture.

35 Gregory Bateson, Une unité sacrée. Quelques pas de plus vers une écologie de l'esprit, Paris, Seuil, [1991] 1996, p. 400-401.

36 Alex Mucchielli, Les sciences de l'information et de la communication, Paris, Hachette, 1995, p. 115. 
jours relation entre des choses (objets, animaux, humains... et bien entendu entre ces diverses catégories entre elles) et que c'est d'abord sur ces choses que le regard se focalise.

Dans sa Critique de la décision, Lucien Sfez, passant au crible les divers auteurs qui abordent la question de la décision et, de façon contiguë, de la communication, dénonce, au-delà des apports de ces " nouvelles approches », non linéaires, non " classiques ", ce tropisme qui conduit les rédacteurs à s'intéresser au « sujet » :

Fonction de ce sujet, la liberté prend alors une connotation morale et métaphysique : intentionnalité, volonté, finalité proposée, moyens de réaliser les fins n'appartiennent qu'à des sujets, c'est-à-dire à des consciences. [...] Toute théorie du sujet est une théorie idéologique dans la mesure où elle justifie, assigne une origine dans la conscience à un déjà-là qu'elle veut cacher $^{37}$.

Ainsi, chez Mucchielli peut-on relever et discuter un positionnement qui conduit à écrire : " cette mise en relation est effectuée par les différents acteurs, chaque interlocuteur d'une situation de communication faisant un travail d'interprétation ou d'inférence ${ }^{38}$ ».

On peut évidemment accepter cette idée que chaque interlocuteur effectue un travail de traduction ou d'inférence, ce qui constitue un progrès très considérable au regard des théories de type shannonnien de la communication en information plus ou moins parfaite. Pourtant, on peut aussi percevoir dans cet extrait une tendance à ramener toute situation de communication à l'interaction entre deux entités séparées : le schéma conceptuel reste proche de celui des interactionnistes et, dans le domaine de la communication, de celui de Shannon dans sa théorie de l'information, à ceci près qu'ici, le récepteur n'est plus passif, mais transforme le message qui lui est transmis. Reste qu'il y a deux émetteurs-récepteurs et un canal de communication à double sens qui les relie.

\footnotetext{
$37 \quad$ Lucien Sfez, Critique de la décision, Paris, Presses de la Fondation nationale des sciences politiques, 1992, p. 351-354. 


\subsection{Oublier les « entités ", spécifier le système de relation}

La proposition de Laflamme s'oppose à une perspective de ce type : parler de relation, dans une perspective communicationnelle, ce n'est pas observer des entités en relation, ce n'est pas rechercher dans ces entités (pour ce qui nous concerne ici, ces " entités » sont des êtres sociaux) les raisons de la communication, de ses formes, de ses évolutions, c'est s'intéresser au système de communication pour en déduire les configurations qui peuvent faire comprendre ces raisons, ces formes, ces évolutions.

L'introduction à son livre Communication et émotion ${ }^{39}$ procède en quatre temps pour nous faire passer de cette conception classique à celle qu'il défend sous l'étiquette de "microsociologie relationnelle " :

1 - «L'émotion et la raison comme inhérentes au sujet » où il montre comment les catégories de raison et d'émotion sont considérées comme ne pouvant provenir que des sujets humains ( l'esprit a pour fondement le sujet ${ }^{40} »$ ).

2 - «La dichotomie de la passion et de la raison " : la foi en la Raison toute puissante s'impose jusqu'à nos jours en laissant percevoir, selon les époques, soit que raison et émotion sont opposées (Aristote, Descartes), soit que l'esprit est à la fois raison et émotion, bien que la raison soit inscrite dans la conscience humaine (Sartre, Kierkegaard), soit que la raison peut et doit maîtriser l'émotion (Freud).

3 - «L'esprit comme entité complexe » : à partir de ce titre, Laflamme développe une autre vision que celle du rationalisme. Celui-ci n'est pas critiquable parce qu'il donnerait une image du réel autre que le réel lui-même. Toute science en est réduite à ce statut, ce qu'elle assume, notamment, par un effort de modélisation.

Désormais, ayant montré que l'on ne peut se prévaloir de la connaissance de la conscience pour y trouver la source de la rationalité qui serait à l'œuvre dans toute communication, notamment son intentionnalité, Laflamme nous conduit vers

39 Simon Laflamme, Communication et émotion..., op. cit.
40 Ibid., p. 15. 
l'idée que "si la conscience peut apparaître comme cause des relations humaines, elle peut aussi se manifester comme leur résultat ${ }^{41}$ ». C'est l'objet du quatrième point de cette introduction.

4 - «Émotion et relation »: «L'étude de l'émotion peut avoir pour fin la simple compréhension des relations humaines. Et cette compréhension peut éviter le détour par la conscience ${ }^{42}$ ». À partir de là est développée une critique du positionnement dualiste des théories dominantes de la communication (l'idée de deux entités reliées par un double canal de relation) par la proposition selon laquelle ce n'est pas la conscience qui crée la relation, mais la relation qui crée la conscience. C'est le contexte relationnel sociohistorique qui détermine la communication, et c'est dans cette communication située que se détermine la conscience. La relation est première et l'on ne peut plus seulement étudier deux êtres en relation pour déterminer leur type de communication. C'est au contraire le système de communication dans lequel baignent les êtres communicants qui permet de comprendre leur type de relation. Dans une telle perspective, la question de l'intentionnalité perd son statut : il n'y a pas forcément intentionnalité dans la relation, la communication n’obéit plus forcément à des finalités posées avant la communication. Bien plus, la question de l'intentionnalité dans la communication n'a plus de sens véritable; dans le système de communication naissent des intentionnalités circonstancielles qui, peut-être guidées par des objectifs préétablis en voie de constante réorganisation, sont elles-mêmes en constante transformation : si le sujet " est toujours un être déjà relationnel ${ }^{43}$ ", il importe moins de connaître les intentions du sujet communicant que de déterminer les particularités du système de relation dans laquelle ce sujet se constitue ${ }^{44}$.

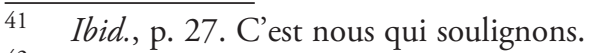

42 Ibid., p. 27.

43 Ibid., p. 31.

44 Il ne s'agit pas d'affirmer qu'il n'existe aucune intentionnalité dans la communication, mais de faire observer que cette intentionnalité n'est ni nécessaire à la communication, ni constamment présente en elle. Dans les conditions
} 
Une telle approche semble féconde pour étudier les situations de communication dans les organisations humaines (ou les relations moins formelles, celles existant au sein d'un couple, par exemple) et comprendre pourquoi ces situations conduisent souvent à des crises.

\section{Principaux éléments du débat}

Il nous semble que le débat porte aujourd'hui sur les points principaux suivants : se passer ou non de l'acteur, accorder la prééminence à l'acteur ou à la relation, examiner le rapport entre l'acteur et la relation, établir le lien entre relation et communication, oser une nouvelle posture sociologique.

\subsection{Peut-on se passer de l'acteur?}

Faut-il et peut-on renoncer à l'acteur pour comprendre les phénomènes communicationnels et, plus largement, sociaux? Parmi les auteurs qui veulent développer les études relationnelles en sociologie, certains, à la manière de Laflamme, considèrent que l'on peut se dispenser de la présence des acteurs dans l'étude des phénomènes sociaux, parce ces derniers sont toujours sous-entendus : ce sont eux qui sont source du social, de diverses manières (par leurs interactions, leurs actions, leurs représentations, leur construction de sens...), tandis qu'ils sont également constitués par le social. Dans le cadre du programme de recherche sur les études de reconstruction, Roth écrit que les sociologues des réseaux sociaux :

exhibent une relation formelle entre des objets de haut et de bas-niveau (macrosociologiques, au niveau du réseau ou système et microsociologiques, au niveau des acteurs) - ils reconstruisent la "structure sociale " qu'ils valident par le biais de descriptions de haut-niveau classiquement admises. En ce sens, ils font l'hypothèse que le bas-niveau choisi (tel un réseau social) contient suffisamment d'information sur le phénomène en question, l'avantage étant souvent que les données de bas-niveau sont

définies ici, pourquoi la querelle de l'intentionnalité aurait-elle lieu, puisqu'une telle position présente au moins l'avantage d'éviter des hypothèses sur la conscience de l'acteur, plus généralement sur sa psyché? 
plus faciles à recueillir et plus robustes ${ }^{45}$.

Ce faisant, ils représentent l'activité des acteurs (ici cognitive) par des graphes et des hypergraphes qui sont la représentation des réseaux sur lesquels ils travaillent et sans perdre les agents de vue, c'est sur les propriétés des treillis qu'ils raisonnent, indépendamment des psychés individuelles ou collectives des acteurs qui les sous-tendent.

D'autres considèrent que la disparition de l'acteur est illégitime parce que c'est en lui que réside la signification du social et que sa présence sous-jacente n'est pas suffisante pour comprendre les phénomènes sociétaux, leur prise en compte à travers une entité globale (réseau, système) s'apparentant à une position holistique et anti humaniste (puisque les valeurs et les affects n'apparaissent pas dans l'analyse).

On peut cependant remarquer que les premiers cités ont malgré tout bien du mal à s'exonérer de l'acteur. Même chez Degennes et Forsé, au-delà des intuitions de la nécessité de sortir d'une sociologie fondée sur des acteurs monadiques, n'y a-t-il pas une même difficulté à envisager une analyse sociologique qui ne soit pas fondée sur les acteurs et l'ouvrage de Degennes et Lemel, Sociologie des comportements intentionnels ${ }^{46}$ n'en est-il pas un signe? De même on a déjà signalé la position de Bajoit qui, tout en appelant à une sociologie relationnelle, écrit par ailleurs que la sociologie ne peut pas ne pas faire du sujet individuel le centre de son analyse.

\subsection{Prééminence de l'acteur ou de la relation?}

L'idée selon laquelle "la relation suppose l'existence d'agents" contrebattue par le fait que « les agents ne se manifestent pas en dehors de relations ${ }^{47}$ " étaye la question précédente et ne paraît pas poser de problème insurmontable; par contre, celle selon laquelle «En ce sens, c'est la relation qui est première et non la

\footnotetext{
$\overline{45}$ Camille Roth, "Reconstruction en sciences sociales... ", op. cit., p. 60.

46 Alain Degenne et Yannick Lemel, Sociologie des comportements intentionnels, Paris, Economica, 2006.

47 Simon Laflamme, Communication et émotion..., op. cit., p. 29.
} 
conscience ${ }^{48}$ " peut être interrogée sur le plan théorique : comme on est dans une boucle de type systémique ni la relation, ni la conscience n'a antériorité ni prééminence. Ceci étant posé, on peut donc décider qu'il est possible pour le chercheur de ne pas considérer la primauté de l'une ou de l'autre des deux catégories, ou encore, qu'il est plus intéressant pour lui de considérer la primauté de la relation plutôt que celle de la conscience.

Il n'y a pas d'issue théorique absolument sûre dans cette querelle. Si l'on adopte la position de Laflamme, on trouve deux idées principales, semble-t-il :

- la conscience n'est pas préexistante à la relation, mais émerge de la relation ${ }^{49}$;

- nous ne possédons aucun moyen de connaître la conscience, pas davantage de la quantifier que de la qualifier.

Dans ces conditions, on a le droit de penser qu'il est formellement plus pertinent et plus efficace pour le chercheur de privilégier l'approche par la relation. De la même façon que dans la métaphore célèbre de la poule et de l'œuf, nous ne saurons jamais où se trouve le point de départ, et celui que nous choisissons est, dans une certaine mesure, arbitraire ${ }^{50}$; l'essentiel (puisque, de toute façon, sur le plan théorique, il est entendu que nous nous plaçons dans la boucle décrite précédemment) est que le point de départ choisi soit plus efficace pour déboucher quelque part, que notre «problématique " soit plus féconde pour la recherche ${ }^{51}$.

$48 \quad$ Ibid., p. 29.

49 Le raisonnement qui suit est valable même si l'on retient la proposition suivante (différente de la proposition énoncée par Laflamme) : « la conscience n'est pas forcément préexistante à la relation et la relation n'est pas forcément préexistante à la conscience. Relation et conscience sont consubstantielles l'une à l'autre ".

50 Pas tout à fait, cependant. Si, avec Laflamme, on considère que la conscience émerge de la relation, on est en droit de considérer, ainsi qu'il vient d'être dit, que le point de départ « relation » est plus pertinent que le point de départ " conscience".

51 Pour faire droit à la critique selon laquelle cette position élimine ou affadit au moins le rôle de l'humain, désincarne l'étude et se rangerait à une position proche de celle de Durkheim (de type holistique), rappelons les propos de Donati selon lesquels " la relation n'élimine pas les termes qu'elle met en connexion; au contraire, elle les met en valeur, les explore, et les exprime ». 
Ce faisant, sur un plan microsociologique, nous nous éloignons des postulats contemporains en matière de théories de la communication sur les deux points suivants : d'une part, de la relation envisagée par ces théories qui est celle qui va de la conscience à la relation, présupposant ainsi que c'est la conscience qui est première et qui induit, crée la communication; d'autre part, du fait que dans cette posture, le chercheur est amené immanquablement à postuler également que c'est le type de conscience, en tant qu'attribut d'un être humain, qui conditionne le type de relation observé.

Au-delà des aspects épistémiques et méthodologiques, le changement de posture proposé par les tenants d'une sociologie relationnelle a aussi des implications pratiques. Ainsi, dans un cas de difficulté communicationnelle au sein d'une organisation, une entreprise, par exemple, ce sont les individus concernés qui sont supposés incapables d'établir un bon système de communication. C'est donc sur ces individus que l'on fera très logiquement porter l'effort de remédiation ${ }^{52}$ : formation, notifications à modifier son comportement, sanctions sont alors les décisions les plus probables. Or, si l'on considère que ce ne sont pas essentiellement les individus (les " consciences ») dans leurs comportements et choix qui génèrent les difficultés, mais que c'est le système de relation qui est principalement en cause dans les dysfonctionnements constatés, les modes de remédiation doivent désormais mettre en lumière prioritairement, non des comportements déviants, mais des comportements relativement adaptés à une configuration particulière d'un système de relation : c'est

Le chapitre XV de Laflamme évoqué plus haut constitue un bel exemple de ce type d'exploration et d'expression des termes mis en connexion.

52 Le Rapport sur la détermination, la mesure et le suivi des risques psychosociaux au travail, de Philippe Nasse et Patrick Légeron, remis au ministre du travail, des relations sociales et de la solidarité, Xavier Bertrand, le 12 mars 2008, est un bon exemple d'une réflexion s'inscrivant dans une approche psychosociale classique, privilégiant les aspects " bio-comportementaux » du stress au travail au détriment d'une approche plus relationnelle et systémique. Position certes intéressante, mais, malgré la dimension ergonomique avancée par les auteurs et la volonté affichée de conjoindre les approches collective et individuelle, logiquement largement centrée sur la psychologie individuelle. 
ce système qu'il faut d'abord interroger et, le cas échéant amender, plutôt que les comportements individuels et collectifs. On peut raisonnablement estimer que si le système relationnel se transforme, les comportements individuels se modifieront également ${ }^{53}$.

Si cette hypothèse est correcte, nous pouvons supposer qu'il y a avantage pour une organisation à focaliser son attention sur ce que l'on considèrera comme la source essentielle du problème (le système de relations) au lieu de concentrer celle-ci sur un problème dérivé (les comportements individuels).

\subsection{La relation est-elle fondatrice de l'être ou l'être de la relation?}

Mais, finalement, que peut signifier l'idée que la relation est fondatrice de l'être?

D'abord, dans une perspective constructiviste, le monde ne nous est pas donné : il est une construction et une projection de notre cerveau, à partir des signes que le monde "réel " inconnaissable lui adresse, qui nous laisse croire que ce monde " extérieur » pénètre en nous (par nos sens, notre appareil cognitif), alors que ce sont nous qui en projetons l'image " autour de nous"(il y a grande difficulté à dire une telle chose $)^{54}$.

53 Bien entendu, le rôle des individus existe et leurs comportements jouent un rôle; mais le parti pris, nous devons le rappeler, est de se focaliser sur le système et non sur ses " parties ». Dans ce sens, une question reste posée, à laquelle les études empiriques devraient apporter une réponse : ces modifications comportementales induites par une modification du système (de communication) seront-elles marginales ou suffisamment importantes pour que l'organisation (ou ses représentants) se disent satisfaits. Il est supposé ici que la réponse à cette question sera " oui » dans un nombre non négligeable de cas.

54 «Pour autant, parce que l'esprit humain fonctionne ainsi, et parce que c'est la seule façon dont nous pouvons en exercer les capacités pour produire de la connaissance scientifique, parce que tout discours et tout lexique catégorisent, il nous faut bien " ponctuer » ce continuum, le catégoriser ("y mettre de l'ordre" , comme dit J.-M. Eloy ici même »), Philippe Blanchet, "Contact, continuum, hétérogénéité, polynomie, organisation "chaotique", pratiques sociales, interventions... Quels modèles? : Pour une (socio) linguistique de la "complexité" ", Cahiers de sociolinguistique, n 8, Rennes, Presses universitaires de Rennes, 2003, http://www.uhb.fr/alc/erellif/credilif/ 
Autrement dit, comme l'écrit Edgar Morin, "notre monde est enfermé dans notre esprit/cerveau, lequel est enfermé dans notre être, lequel est enfermé dans notre monde ${ }^{55}$ ". Ainsi, il n’y a pas relation entre deux entités préexistantes et objectivement séparées, l'être et le monde, mais unité principielle entre l'être et le monde, chacun n'ayant de sens, d'existence, que par l'autre. Couper la boucle, ici aussi, conduit à ne plus comprendre, à abandonner la raison complexe pour adopter une raison linéaire et tronquée ${ }^{56}$.

Ensuite, dans une telle conception, isoler les éléments de leur relation n'a plus de sens, puisque l'élément n'existe que parce qu'il y a relation, tout comme la relation suppose l'existence des éléments. Une métaphore du physicien anglais David Bohm nous semble expliciter assez clairement cette idée. Bohm écrit que :

Ce qui est considéré comme premier, c'est la complétude, et les parties sont considérées comme secondaires, dans le sens que leur nature et leur activité ne peuvent être appréhendées qu'à la lumière du tout. Cela pourrait se résumer par le principe suivant : la complétude du tout et de ses parties. Et par le principe inverse : la " partitude » des parties et du tout. Les deux principes sont valables. Mais je dis qu'il faut insister sur le principe de la complétude du tout et des parties ${ }^{57}$.

et que :

CdS8-2003.pdf, site consulté le 29 septembre 2008.

Edgar Morin, La Méthode, tome 3, La connaissance de la connaissance, Paris, Seuil, 1986, p. 214.

On trouve cette idée chez Gregory Bateson (Une unité sacré..., op. cit.). La même idée se retrouve également, entre autres, chez Joël de Rosnay (Le macroscope. Vers une vision globale, Paris, Seuil, Paris, 1975, p. 120-121). Voir aussi l'idée selon laquelle si on " coupe la boucle ", c'est plutôt en grossissant un aspect de celle-ci pour l'observer plus commodément : "La notion de focalisation que propose D. Robillard ici même à la fois pour expliciter un regard "disciplinaire" et une catégorisation, me semble, à cet égard tout à fait adaptée. Catégoriser plutôt que "découper" (donc disjoindre), c'est alors opérer un "zoom avant" qui établit un segment du continuum en objet grossi, le distingue provisoirement $\mathrm{du}$ reste du contexte continu, et permet de l'examiner en détail. Mais il reste à opérer ensuite le "zoom arrière" [...] qui permet de réinsérer le segment dans le continuum, dans le contexte global, donc de le relativiser et de le mettre en perspective " (Philippe Blanchet, "Contacts, continuum, hétérogénéité, polynome, organisation " chaotique ", pratiques sociales, interventions...,op. cit.).

David Bohm, La danse de l'esprit ou le sens déployé, La Varenne-Saint Hilaire, Séveyrat, 1985, p. 33. C'est nous qui soulignons. 
La fragmentation est donc une attitude mentale qui prédispose l'esprit à voir les séparations entre les choses comme absolues et définitives, plutôt que comme le résultat d'un mode de pensée n'ayant qu'une utilité et une validité relatives et limitées ${ }^{58}$.

On trouve également chez d'autres auteurs, par exemple Elias, l'intuition de ce que nous dit Laflamme. Bien qu'il reste en deçà de la position de Laflamme lorsqu'il affecte les limites de l'autonomie des humains à leurs origines sociales, familiales, Elias écrit cependant :

Et c'est l'ordre de ce réseau permanent [le « réseau permanent de besoins, une perpétuelle alternance de désirs et de satisfaction, de prendre et de donner ", NDA] qui ne commence nulle part, c'est l'histoire de ses relations qui font la nature et la forme de la personne individuelle. Même la nature et la forme de sa solitude, même ce que l'individu ressent comme son "intériorité » reçoivent l'empreinte de cette histoire - l'empreinte du filet de relations humaines dont il est l'un des nœuds et au sein duquel il vit et accède à son individualités9.

\subsection{Relation et communication : deux concepts identiques?}

Donati considère que réduire les relations à la communication correspond à une forme de "relationnisme " qui lui semble fautif :

Dans la postmodernité, la dialectique du sujet et de l'institution, de l'action et de la structure, etc., suit le simple déploiement des relations conçues en général comme des flux de communication. Le code symbolique interprétant ce processus est essentiellement communicationnel : les relations sont englobées en tant que communication et sont de ce fait réduites à n'être que cela ${ }^{60}$.

Dans son essai Communication et émotion, Laflamme considère plutôt qu'une société ne peut être si elle n'est pas communicationnelle, s'appuyant sur Sfez : "Que signifie "société de communication", par laquelle se désigne elle-même notre société aujourd'hui? Se désignant ainsi, la société ne dit rien de plus que : "je suis société". Elle réaffirme le lien qui unit ses parties entre

$58 \quad$ Ibid., p. 35.

59 Norbert Elias, La société des individus, op. cit., p. 72.

60 Pierpaolo Donati, La relation comme objet..., op. cit., p. 10. 
elles. La palissade, si l'on veut, ou encore tautologie ${ }^{61}$ ». Malgré la préférence que nous marquons ${ }^{62}$ pour la position de Laflamme, il nous semble que sur la controverse autour des termes " relation " et " communication ", les deux auteurs devraient dialoguer pour clarifier leurs positions respectives qui nous semblent brouillées du fait même de la proximité de leurs positions sur les autres points.

Pour en terminer, lisons encore une fois Laflamme :

La définition d'un acteur rationnel est étonnante. La persistance de cette position dans la sociologie l'est davantage. L'étonnement vient ici de ce que la définition ne correspond pas à l'expérience et à l'observation du vécu humain. Que l'humain soit capable de rationalité, cela ne fait aucun doute. Que l'humain soit essentiellement rationnel - notion à laquelle se greffent celles de conscience, d'intérêt, d'intention, de liberté - cela est indéfendable. [...] Bien sûr, à nouveau, l'humain est capable de gestes intentionnels; mais tout ce que fait une personne dans une journée, tout ce qu'elle dit n'est pas aménagé par une intention. Comment peut-on avoir l'intention d'aimer quelqu'un [...] de se mettre en colère à la lecture d'un article de journal, de manifester son inquiétude à l'égard de la question que pose une personne que l'on croise sur son chemin? Toute cette sociologie a pour point de départ un acteur social qui vit en dehors des relations, une monade, une conscience qui

61 Lucien Sfez, Critique de la communication, Paris, Seuil, Points, [1988] 1992, dans Simon Laflamme, Communication et émotion..., op. cit., p. 37, note 2.

Dans Communication et émotion, l'objectif de Laflamme est de montrer, à partir d'une analyse "microsociale ", que le sujet ne peut apparaître comme le centre de l'analyse sociologique : c'est le concept de relation qui est ici au centre. Il en donne la justification dans le chapitre XV, « Â propos de l'amour ", notamment en écrivant, p. 173 : "L'amour est le milieu de la communication le plus complet qui puisse se concevoir. Entre les partenaires se crée un champ informatif intégral, un champ baigné de savoir-sensation. Le champ se couvre des partenaires qui, tout à la fois, se glissent en lui et le constituent. Le champ est milieu amoureux et être de l'amour. Les partenaires ne se communiquent pas des informations, ils sont eux-mêmes ces informations. L'enjeu de ce qui s'échange entre eux est leur être respectif et leur être conjugué ». N'est-ce pas une belle manière de dire que la communication considérée comme l'incarnation de la relation (mais pas de façon substantivée) est bien (en tant que " champ ») l'origine des émotions et de la situation amoureuse? Ne peut-on penser, également, que ce passage exprime de façon claire ce qu'écrit Donati : " tout objet sociologique doit être défini comme une relation sociale de manière sensible, sachant que l'objet de la sociologie est la relation sociale "incarnée" » (op. cit., p. 12). 
réfléchit en elle-même, à tout moment, en s'extrayant du monde, elle parle d'un acteur social qui n'est pas toujours en société et qui n'est pas en action. En insistant comme elle le fait sur la rationalité, sur la conscience et sur l'intention, cette sociologie déshumanise l'objet dont elle entend faire l'apologie. L'humain est capable de raison et d'intention; mais il est aussi capable de bien d'autres choses ${ }^{63}$.

Et encore :

On a ici affaire à un double résultat de l'histoire de la sociologie, à une antinomie nécessaire : les acteurs sociaux sont déterminés par les structures sociales et déterminent ces structures, dans une relation dialectique. Ce paradoxe a désormais valeur de postulat; il n'est plus de l'ordre du débat sociologique ${ }^{64}$.

Pour Laflamme, comme pour Donati, l'approche relationnelle permet aujourd'hui de dépasser les impasses et les insuffisances des théories dominantes en sociologie. Que ces théories aient apporté des éclairages intéressants, voire fondamentaux, à la compréhension des systèmes sociaux, cela est indéniable et les références qu'égrènent les auteurs au long de leurs textes montrent à quel point ils en sont conscients.

\subsection{Postulat et pari}

Contrairement à ce que semble penser Hirshhorn dans l'article déjà cité, il n'est pas si probable que l'avenir de la sociologie française se joue autour de la question du sujet. Hirshhorn écrit :

Pourtant, ce à quoi nous assistons est bien autre chose que le développement de paradigmes concurrentiels, c'est un enrichissement ou un assouplissement de l'actionnisme sous l'effet de la rencontre avec des paradigmes voisins, mais quelque peu différents. Ainsi la théorie de la structuration de Giddens ne constitue pas une remise en cause du paradigme actionniste. Elle conduit seulement à insister sur des dimensions qui, tout en étant présentes, étaient moins soulignées, comme par exemple celle du maintien et la transformation des structures à travers l'action des individus ou à élargir la notion de rationalité en faisant place aux notions de compétence et de routine. Toutefois, émerge dans la

63 Simon Laflamme, Suites sociologiques, Sudbury, Prise de parole, coll. "Epistémè », 2006, p. 117-118.

64 Ibid., p. 84. 
sociologie française, et c'est peut-être autour de cette question que se situeront dans les années qui viennent les véritables débats, une sociologie du "sujet ", référée entre autres à G. H. Mead (1963), à A. Schütz (1987) et plus près de nous P. Berger et T. Luckmann (1986), c'est-à-dire l'introduction d'une démarche proprement phénoménologique. Celle-ci n'est évidemment pas incompatible avec le paradigme actionniste, mais elle constitue cependant une perspective différente, car on ne peut réduire le "sujet " à l'acteur rationnel. Si le "sujet " est au centre de l'analyse, le contenu des questions change. Il ne porte plus seulement sur le sens que l'acteur donne à ses comportements, mais sur la construction sociale du soi, sur les modes d'élaboration de l'identité. Bien entendu, elles ne constituent pas le seul objet de la sociologie et la compréhension de la rationalité des acteurs en situation reste essentielle, y compris pour y répondre. Elles n'en représentent pas moins un élargissement de la perspective adoptée qui pose peut-être à terme le problème d'un remaniement du paradigme actionniste ou même celui d'une redéfinition de l'objet de la sociologie ${ }^{65}$.

Cette longue citation paraît utile pour que soit conservée la précision du propos de l'ancienne présidente de l'Association Internationale des Sociologues de Langue Française.

À ce propos répond, par la négative, celui de Laflamme. Son interprétation d'une sociologie relationnelle veut, au contraire, évacuer toute position phénoménologique, tout approfondissement de la notion de rationalité qui puisse permettre de comprendre les comportements de l'acteur, lesquels permettraient d'expliquer le social émergent et, dans une optique interactionniste, second, dans la temporalité comme dans la méthode.

S'il faut approfondir la rationalité, ce n'est qu'en admettant que celle-ci est une "émorationalité ", ce qui signifie l'existence à la fois d'émotion et de raison qui ne prennent naissance que dans la relation et la communication.

"Tirer les conséquences de cette importante conclusion ", ne peut se faire, pour Laflamme :

qu'en faisant de l'humain lui-même le lieu de la dialectique, c'est-à-dire, et en ce sens Habermas aura eu raison, qu'on ne peut comprendre l'humain que comme relationnel et, en tant qu'humain, comme communicationnel $[\ldots]$ il faut aller au-delà des concepts de structure,

65 Monique Hirshhorn, "L'actionnisme ", op. cit., p. 57. 
d'agent, d'acteur ou de sujet. Il faut aller dans l'échange, sans partir ni de la structure de la relation ou de la structure dans laquelle se déroule la relation, ni des agents qui sont en relation; il faut partir de la relation même ${ }^{66}$.

Cette posture a des conséquences en termes de méthode de recherche, notamment de modélisation.

Étudier une situation de communication ou, plus généralement, une situation sociétale par le biais d'un système que l'on construit et que l'on spécifie en en mesurant certaines dimensions n'est pas un retour vers quelque holisme méthodologique durkheimien ou non. Cette situation est décrite dans une fic$\operatorname{tion}^{67}$, un " modèle " qui, nous faisant monter en abstraction, loin d'obscurcir le fait et le propos, éclaire l'un et l'autre. Le langage ainsi conçu permet de dire ce qui semblait indicible, d'ordonner ce qui paraissait chaotique, d'apporter du sens, d'objectiver ce qui n'est jamais, hors cela, que notre représentation d'une partie du monde.

C'est à ce prix, celui d'une plus grande abstraction dans la réflexion, que l'on pourra rendre nos représentations plus complexes, prendre en compte les contradictions et les paradoxes et mettre en scène un social constitué de la relation dans le champ sociohistorique.

En construisant un modèle relationnel qui semble ignorer l'acteur en tant que tel et qui privilégie l'étude de l'état du système, ce que nous appelons, après Elias, la configuration du système, nous essayons de comprendre comment donner une interprétation de ce que nous pouvons observer "sur le terrain », comme dit le sociologue.

Le système n'est alors qu'une "machine à problème ", selon les termes si bien adaptés d'Yves Barel ${ }^{68}$ et il peut permettre de modifier l'approche, de poser autrement le problème. En parti-

66 Simon Laflamme, Communication et émotion, op. cit., p. 143-145.

67 Belle phrase de Léonard de Vinci : " Je créerai une fiction qui exprimera de grandes choses ", (Les carnets de Léonard de Vinci 2, Paris, Gallimard, 1942, p. 443) et aussi, trait lumineux de Valéry : "L'arbitraire créant le nécessaire " (Euvres I, Paris, Gallimard, Bibliothèque de la Pléiade, 1957, p. 1181).

68 Yves Barel, «L'idée de système dans les sciences sociales », Esprit, n 1, 1977, p. 76. 
culier, nous retrouvons dans cette démarche l'injonction de l'auteur du Paradoxe et le système, qui suggérait (il y a déjà trente ans) que : " il se pourrait que les vrais systèmes vivants, à la différence des systèmes de chambre, régulent leurs rapports avec l'extérieur moins par une politique d'“importation" que par des modifications internes, des modifications structurales du systeme $e^{69}$ ".

Voilà le postulat et le pari.

Postulat, l'idée de la nécessité d'interroger des situations sociétales à l'aide d'un appareillage qui gomme apparemment l'acteur au profit d'une caractérisation de la situation en ellemême.

Pari, celle selon laquelle on peut mettre en lumière les « modifications structurales " du système qui décrit la situation étudiée et relier ainsi les phénomènes observés à ces modifications.

Procéder ainsi semble de nature à améliorer la compréhension du social et les procédures de remédiation face aux crises et difficultés rencontrées par nos systèmes sociétaux, particulièrement en matière de communication.

\section{Bibliographie}

Bajoit, Guy, Pour une sociologie relationnelle, Paris, PUF, 1992.

Bajoit, Guy, Contribution à une sociologie du sujet, Paris, L'Harmattan, 2000.

Bajoit, Guy, Le changement social. Approche des sociétés occidentales contemporaines, Paris, Armand Colin, coll. "Cursus », 2003.

Barel, Yves, "L'idée de système dans les sciences sociales ", Esprit, $\mathrm{n}^{\circ} 1$, 1977, p. 69-82.

Bateson, Gregory, Une unité sacrée. Quelques pas de plus vers une écologie de l'esprit, Paris, Seuil, [1991] 1996.

Blanchet, Philippe, "Contact, continuum, hétérogénéité, polynomie, organisation "chaotique", pratiques sociales, interventions... Quels modèles? : Pour une (socio)linguistique de la "complexité" ", Cahiers

69 Ibid., p. 77. C'est nous qui soulignons. 
de sociolinguistique, $\mathrm{n}^{\circ}$ 8, Rennes, Presses universitaires de Rennes, 2003, http://www.uhb.fr/alc/erellif/credilif/CdS8-2003.pdf, site consulté le 29 septembre 2008.

Bertalanffy, Ludwin von, Théorie générale des systèmes, Paris, Dunod, 1968.

Bohm, David, La danse de l'esprit ou le sens déployé, La Varenne-Saint Hilaire, Séveyrat, 1985.

Boudon, Raymond, Etudes sur les sociologues classiques, Paris, PUF, 1998.

Boudon, Raymond, La logique du social. Introduction à l'analyse sociologique, Paris, Hachette, 1979.

Boudon, Raymond et François Bourricaud, Dictionnaire critique de la sociologie, PUF, [1982] 1994.

Degenne, Alain et Michel Forsé, "Analyse de réseau et structuralisme ", dans Jean-Michel Berthelot (dir.), Sociologie. Épistémologie d'une discipline. Textes fondamentaux, de Boeck, 2000, p. 221-234.

Degenne, Alain et Michel Forsé, Les réseaux sociaux. Une analyse structurale en sociologie, Paris, Armand Colin, coll. «U », 1994.

Degenne, Alain et Yannick Lemel, Sociologie des comportements intentionnels, Paris, Economica, 2006.

Donati, Pierpaolo, "La relation comme objet spécifique de la sociologie ", Revue du MAUSS, 2, n 24, 2004, http://www.cairn.info/article_p. php?ID_ARTICLE=RDM_024_0233, site consulté le 25 septembre 2008.

Elias, Norbert, La société des individus, Paris, Arthème Fayard, [1987] 1991.

Emirbayer, Mustafa, "Manifesto for a Relational Sociology ", American Journal of Sociology, vol. 103, n 2, 1997, p. 281-317.

Grossetti, Michel et Frédéric Godart, "Réseaux et histoires ", Sociologies, 17 octobre 2007, http://sociologies.revues.org/document 240.html, site consulté le 25 septembre 2008.

Hirshhorn, Monique, «L'actionnisme », dans Jean-Michel Berthelot (dir.), La sociologie française contemporaine, Paris, PUF, [2000] 2001, p. 47-58.

Laflamme, Simon, Communication et émotion. Essai de microsociologie relationnelle, Paris, L'Harmattan, coll. "Logiques sociales », 1995.

Laflamme, Simon, Suites sociologiques, Sudbury, Prise de Parole, coll. "Epistémè ", 2006.

Morin, Edgar, La Méthode, tome 3, La connaissance de la connaissance, Paris, Seuil, 1986.

Mucchielli, Alex, Les sciences de l'information et de la communication, Paris, Hachette, 1995. 
Nasse, Philippe et Patrick Légeron, Rapport sur la détermination, la mesure et le suivi des risques psychosociaux au travail, remis au ministre du travail, des relations sociales et de la solidarité, Xavier Bertrand, le 12 mars 2008.

Rosnay, Joël de, Le macroscope. Vers une vision globale, Paris, Seuil, 1975.

Roth, Camille, "Reconstruction en sciences sociales : le cas des communautés de savoir ", Nouvelles Perspectives en Sciences Sociales, vol. 2, $\mathrm{n}^{\circ}$ 2, 2007, p. 59-101.

Sfez, Lucien, Critique de la décision, Paris, Presses de la Fondation nationale des sciences politiques, 1992.

Sfez, Lucien, Critique de la communication, Paris, Seuil, Points, [1988] 1992.

Valéry, Paul, Euvres I, Paris, Gallimard, Bibliothèque de la Pléiade, 1957.

Vinci, Léonard de, Les carnets de Léonard de Vinci 2, Paris, Gallimard, 1942.

Watzlawick, Paul, Janet Helmick Beavin et Don D. Jackson, Une logique de la communication, Paris, Seuil, [1967] 1971.

Weber, Max, "Lettre à R. Liefman le 9 mars 1920 ", cité dans Raymond Boudon et François Bourricaud, Dictionnaire critique de la sociologie, Paris, PUF, [1982] 1994, p. 1, article «Action ». 